\title{
An investigation of the Peutz-Jeghers gene (LKB1) in sporadic breast and colon cancers
}

\author{
L F Forster, S Defres, D R Goudie, D U Baty, F A Carey
}

\begin{abstract}
Aims-To explore the role of the PeutzJeghers gene (LKB1) in sporadic breast and colon cancers.

Methods-Thirty consecutive sporadic carcinomas of the breast and 23 of the colon were selected. DNA was extracted from paraffin wax embedded tissue and analysed for loss of heterozygosity (LOH) at microsatellite markers D19S886 and D19S565 close to the LKB1 gene. Tumours showing LOH were screened for LKB1 mutations by single strand conformational polymorphism (SSCP).

Results-Five breast carcinomas showed LOH $(21 \%$ and $7 \%$ of those informative for D19S886 and D19S565, respectively). Five of the colorectal carcinomas showed LOH (15\% and $36 \%$ of those informative for D19S886 and D19S565, respectively), with one sample showing allele loss with both markers. Screening of these 10 carcinomas by SSCP identified one migrational shift but sequencing revealed an intronic polymorphism only. Therefore, no coding mutations were found in these carcinomas. Conclusions-These findings suggest that although allele loss at the LKB1 locus occurs relatively frequently in sporadic breast and colon cancers, mutations do not seem to be a feature.

(f Clin Pathol 2000;53:791-793)
\end{abstract}

Keywords: Peutz-Jeghers syndrome; LKB1; colon cancer; breast cancer

First reported in the literature in 1921 by Peutz ${ }^{1}$ and further characterised by Jeghers et al in $1949,{ }^{2}$ Peutz-Jeghers syndrome is a rare, autosomal dominant condition with near complete penetrance. It is manifest clinically by the development of mucocutaneous pigmentation and morphologically characteristic gastrointestinal hamartomous polyps.

Initially believed to run a benign course, it

Departments of Pathology and Genetics, Ninewells Hospital and

University of Dundee, DD1 9SY, Scotland,

UK

L F Forster

$S$ Defres

D R Goudie

D U Baty

F A Carey

Correspondence to:

Dr Forster

email:

lornef@tuht.scot.nhs.uk

Accepted for publication 7 January 2000

has become increasingly apparent since the 1960 s that the syndrome is associated with an increased incidence of malignancy, including cancers of the breast, gut, pancreas, ovary, and endometrium. In addition, the malignancies appear to develop at a younger age in those with Peutz-Jeghers syndrome than in the general population. ${ }^{3}$ Furthermore, two rare tumours, sex cord tumour with annular tubules and adenoma malignum of cervix, have been associated with the syndrome, with up to $10 \%$ of all cases of adenoma malignum occurring in patients with Peutz-Jeghers syndrome. ${ }^{4}$ This increased risk was quantified in 1987 by Giardiello and co-workers, who reported an 18-fold increase in the risk of cancer development in
Peutz-Jeghers syndrome compared with the general population. ${ }^{3}$ This finding has been refined to give relative risks of 18.5 in women and 6.2 in men, with the relatively greater risk in women being attributable to the development of breast and gynaecological malignancy. ${ }^{5}$

Using comparative genomic hybridisation and linkage analysis techniques, the PeutzJeghers gene (LKB1) has been mapped to the short arm of chromosome 19 at $19 \mathrm{p} 13.3 .{ }^{6}{ }^{7} \mathrm{It}$ has been suggested that a second locus on the long arm of the same chromosome is responsible for the syndrome in a minority of patients. ${ }^{8}$ The LKB1 gene encodes a serine threonine kinase (STK11). ${ }^{9}$ Several mutations of LKB1 have been reported that, in the main, lead to truncations of the protein and resultant loss of enzyme activity. It is this loss of enzyme activity that is believed to be responsible for the Peutz-Jeghers syndrome phenotype. Loss of heterozygosity studies of the hamartomas and adenocarcinomas occurring in patients with Peutz-Jeghers syndrome ${ }^{6}{ }^{10}$ have identified loss of the wild-type allele, thus conforming with Knudson's two hit hypothesis of tumorigenesis, and suggesting that the Peutz-Jeghers gene is a tumour suppressor gene. ${ }^{11}$ Interestingly, PeutzJeghers syndrome is the first cancer susceptibility syndrome that occurs as a result of inactivation of a protein kinase.

Tumour suppressor genes identified in other cancer syndromes (such as familial adenomatous polyposis) are frequently implicated in sporadic cancers. In this context, the aim of our study was to explore the role of LKB1 in sporadically occurring breast and colon cancers. Breast and colon cancers were chosen because they occur with relative frequency in both Peutz-Jeghers syndrome and in the general population. The tumours were screened for loss of heterozygosity ( $\mathrm{LOH}$ ) at $19 \mathrm{p} 13.3$ using microsatellite markers and those showing $\mathrm{LOH}$ were analysed for mutations of the remaining allele.

\section{Methods}

Thirty consecutive, sporadic carcinomas of the breast and 23 of the colon treated by surgical excision in Dundee, Scotland in 1997 were included in our study. In each case, tumour tissue was matched with normal tissue from the same specimen, usually an uninvolved lymph node. DNA was extracted from paraffin wax (Hybaid, Ashford, Middlesex, UK) and amplified by the polymerase chain reaction (PCR) using the microsatellite markers D19S886 and D19S565. These markers have been shown previously to map closely to the Peutz-Jeghers syndrome locus. ${ }^{6}$ The reverse primers were embedded tissue using a commercial kit 


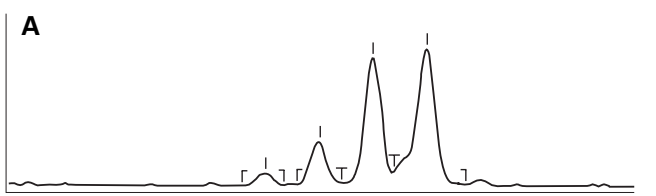

B

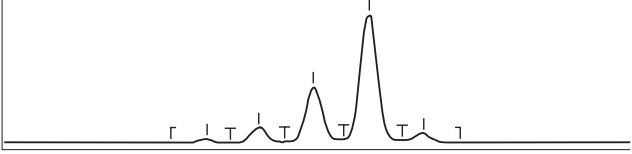

Figure 1 Loss of heterozygosity in a breast carcinoma. (A) Normal tissue: two tall peaks representing the two different normal alleles. (B) Breast carcinoma tissue: owing to loss of an allele (loss of heterozygosity), the second tall peak (far right) is missing.

labelled fluorescently-D19S886 with Fam and D19S565 with Hex. Amplification of D19S886 was initiated by hot start for five minutes at $94^{\circ} \mathrm{C}$, followed by 35 cycles of one minute at $94^{\circ} \mathrm{C}, 30$ seconds at $50^{\circ} \mathrm{C}$, and 30 seconds at $72^{\circ} \mathrm{C}$. The conditions for D $19 S 565$ were similar apart from an annealing temperature of $55^{\circ} \mathrm{C}$. The amplified products were resolved by electrophoresis on an agarose gel ( $1.5 \% \mathrm{wt} / \mathrm{vol}$ in $0.5 \times$ Tris borate EDTA buffer) containing ethidium bromide $(0.5 \mathrm{mg} / \mathrm{ml})$ and viewed under UV light. LOH was detected using the ABI 310 fragment analyser, comparing normal and tumour samples in each case.

Those samples showing LOH were selected for mutation analysis. DNA extracted from paraffin wax embedded tissue using standard techniques was amplified by PCR (initiated by hot start for 15 minutes at $94^{\circ} \mathrm{C}$, followed by 35 cycles of one minute at $94^{\circ} \mathrm{C}, 45$ seconds at $64^{\circ} \mathrm{C}$, and 30 seconds at $72^{\circ} \mathrm{C}$ ). Primers were selected to cover the nine exons of LKB1. ${ }^{12}{ }^{13}$ The amplified products from each exon were screened by single strand conformational polymorphism (SSCP) analysis at room temperature on $10 \%$ polyacrylamide gels (ratio, $49: 1$ ) containing $10 \%$ glycerol. Any sample showing a migration shift compared with the normal control was sequenced.

\section{Results}

Nineteen of the 30 breast and 11 of the 23 colon carcinomas were informative for D19S886. Of these, four breast and four colon carcinomas showed LOH (fig 1). With the marker D19S565, one of 15 informative breast carcinomas and two of 13 informative colon carcinomas showed LOH. One colon carcinoma showed $\mathrm{LOH}$ with both markers. Thus, we identified 10 carcinomas showing allele loss at the Peutz-Jeghers syndrome locus. Of these,

Table 1 Allele loss and mutation rates for different populations

\begin{tabular}{lllllll}
\hline Population & Carcinoma & Number & LOH & Marker & Mutations & \multirow{2}{*}{ Ref } \\
\hline UK & Breast & 62 & $3 / 40$ & D19S565 & Nil & 12 \\
Norwegian & Colorectal & 75 & $13 / 50$ & D19S886 & Nil & 15 \\
Italian & Colorectal & 71 & $10 / 52$ & D19S886 & 1 & 16 \\
Korean & Colorectal & 23 & $9 / 17$ & D19S886 & 5 & 13 \\
& & & $6 / 15$ & D19S883 & 4 & \\
UK & & & $6 / 22$ & D19S565 & 3 & Current \\
& Breast & \multirow{2}{*}{20} & $1 / 15$ & D19S565 & Nil & \\
& & & $4 / 19$ & D19S886 & & \\
& Colorectal & 23 & $2 / 13$ & D19S565 & Nil & \\
& & & $4 / 11$ & D19S886 & & \\
\hline
\end{tabular}

$\mathrm{LOH}$, loss of heterozygosity, given as allele loss/number informative. only one sample (a colon carcinoma) showed a band shift on SSCP analysis. Sequencing revealed a $\mathrm{T}$ to $\mathrm{C}$ transition but this occurred in an intronic region not crucial for splicing and is therefore likely to be non-coding and of no pathogenetic importance.

\section{Discussion}

The existence of cancer associated syndromes provides direction for the study of sporadic cancers. Accumulating epidemiological evidence suggests that Peutz-Jeghers syndrome predisposes to cancer development, at sites both within and outside the gastrointestinal tract. There is increasing interest in a "hamartomaadenoma-carcinoma" sequence to explain the development of cancer in patients with PeutzJeghers syndrome. ${ }^{14}$ However, many of the malignancies occur at sites outside the gastrointestrinal tract and those occurring within do not necessarily arise from hamartomas.

The recent literature contains several studies that explore the role of the Peutz-Jeghers syndrome gene in sporadic cancers; in particular, looking for allele loss and evidence of mutations at this locus. The results show some inconsistences. Most studies show a notable frequency of allele loss but mutational rates are variable between populations (table 1). No mutations were found in a UK study of 62 sporadic breast cancers, ${ }^{12}$ none in a study from Norway of 75 colon cancers, ${ }^{15}$ and only one in an Italian series of 71 colorectal cancers. ${ }^{16}$ However, a study from Korea gives contradicting results, identifying seven mutations in 23 colorectal carcinomas. ${ }^{13}$ Interestingly, these workers separated their series into right and left sided colorectal carcinomas and found that the mutations were present in the left sided carcinomas only (seven of 13). This said, their incidence of mutations is still higher than that reported elsewhere without taking anatomical segregation into account. Why this study should be different from the others remains unexplained, but it could be related to the different incidence of colorectal carcinoma in Korea and to population differences, with all the other studies having been performed on Western populations. Other studies of testicular and gastric cancers have found similar results to the Western studies. ${ }^{15}{ }^{17}$ Indeed, a recent large study of 129 cancers from a variety of sites identified only three mutations. ${ }^{18}$

Our findings are in keeping with other work on Western populations and with the only previous UK study on breast cancers. To date, similar work has not been reported on colon cancers in a UK population. No mutations were detected in our study. However, we did identify an intronic variation, confirming that our methods were capable of detecting sequence changes. It could be that mutations are occurring at the LKB1 locus that cannot be detected by SSCP analysis - for example, large genomic rearrangements. Alternatively, it is possible that other mechanisms such as promoter methylation are occurring as the "second hit" phenomenon.

Thus, although it seems that allele loss at LKB1 in sporadic breast and colon cancers is a 
relatively frequent event, mutations at this locus appear not to be a feature. Hemizygosity for LKB1, even in the absence of a mutation, may in itself be deleterious. Alternatively, another as yet unspecified gene might be involved.

1 Peutz JL. On a very remarkable case of familial polyposis of the mucous membrane of the intestinal tract and nasopharynx accompanied by peculiar pigmentation of the skin and mucous membrane. Ned Tijdschr Geneeskd 1921;10:134-46.

2 Jeghers H, McKusick VA, Katz KH. Generalised intestinal polyposis and melanin spots of the oral mucosa, lips and digits. N Engl F Med 1949;241:992-1005.

3 Giardiello FM, Welsh SB, Hamilton SR, et al. Increased risk of cancer in the Peutz-Jeghers syndrome. N Engl $7 \mathrm{Med}$ 1987;316:1511-14

4 Srivatsa PJ, Keeney GL, Podraiz KC. Disseminated cervical adenoma malignum and bilateral ovarian sex cord tumours with annular tubules associated with Peutz-Jeghers synwith annular tubules associated with
drome. Gynecol Oncol 1994;33:256-64.

5 Boardman LA, Thibodeau SN, Schaid DJ, et al. Increased risk for cancer in patients with the Peutz-Jeghers syndrome. risk for cancer in patients with the

6 Hemminki A, Tomlinson I, Markie D, et al. Localization of a susceptibility locus for Peutz-Jeghers syndrome to $19 \mathrm{p}$ using comparative genomic hybridization and targeted linkage analysis. Nat Genet 1997;15:87-90.

7 Amos CI, Bali D, Thiel TJ, et al. Fine mapping of a genetic locus for Peutz-Jeghers syndrome on chromosome $19 \mathrm{p}$. Cancer Res 1997;57:3653-6.
8 Mehenni H, Blouin JL, Radhakrishna U, et al. Peutz-Jeghers syndrome: confirmation of linkage to chromosome and identification of a potential second locus, on 19q13.4. Am 7 Hum Genet 1997;61:1327-34.

9 Jenne DE, Reiman H, Nezu JI, et al. Peutz-Jeghers syndrome is caused by mutations in a novel serine threonine kinase. Nat Genet 1998;18:38-44.

10 Gruber SB, Entius MM, Petersen GM, et al. Pathogenesis of adenocarcinoma in Peutz-Jeghers syndrome. Cancer Res 1998;58:5267-70.

11 Knudson AG. Antioncogenes and human cancer. Proc Natl Acad Sci U S A 1993;90:10914-21.

12 Bignell GR, Barfoot R, Seal S, et al. Low frequency of somatic mutations in the LKB1/Peutz-Jeghers syndrome gene in sporadic breast cancer. Cancer Res 1998;58:1384-6.

13 Dong SM, Kim KM, Kim SY, et al. Frequent somatic mutations in serine/threonine kinase 11/Peutz-Jeghers syndrome gene in left-sided colon cancer. Cancer Res 1998;58:378790

14 Wang Z-J, Ellis I, Zauber P, et al. Allelic imbalance at the LKB1 (STK11) locus in tumours from patients with Peutz-Jeghers syndrome provides evidence for a hamartoma-(adenoma)-carcinoma sequence. $f$ Pathol 1999;188:9-13.

15 Avizienyte E, Roth S, Loukola A, et al. Somatic mutations in LKB1 are rare in sporadic colorectal and testicular tumours. Cancer Res 1998;58:2087-90.

16 Resta N, Simone C, Mareni C, et al. STK11 mutations in Peutz-Jeghers syndrome and sporadic colon cancer. Cancer Peutz-Jeghers syndrome

17 Park WS, Moon YW, Yang YM, et al. Mutations of the STK11 gene in sporadic gastric carcinoma. Int $\mathcal{F}$ Oncol 1998;13:601-4

18 Avizienyte E, Loukola A, Roth S, et al. LKB1 somatic mutations in sporadic tumors. Am f Pathol 1999;154:677-81.

\section{WHAT'S YOUR VIEW?}

\section{Pathology Interactive: useful or useless?}

To make Pathology Interactive a more useful tool to help you in your CPD

... we need your views

Please take time to complete a self completion questionnaire and fax it back to us

Questionnaire available for printing out on:

Pathology Interactive CD Vol 1 issue3 (Dec 1999)

Pathology Interactive CD Vol 22000

fournal of Clinical Pathology website - www.jclinpath.com

Fax completed forms to Natalie Davies, Editorial Assistant, Journal of Clinical Pathology (+44 (0)20 7383 6668).

Your time and help are appreciated. We especially thank all those ACP members who returned questionnaires circulated in the September 1999 journal issue for their valuable feedback.

Pathology Interactive 2000 offers up to three CPD credits per article; later issues feature case study and picture quiz formats 